\title{
EFICIENCIA DE REABSORCIÓN DE NITRÓGENO Y FÓSFORO Y SUS RELACIONES ESTEQUIOMÉTRICAS DURANTE LA SENESCENCIA EN ESPECIES DEL DESIERTO SONORENSE \\ NITROGEN AND PHOSPHORUS REABSORPTION EFFICIENCY AND STOICHIOMETRIC RELATIONSHIPS DURING SENESCENCE IN SPECIES OF THE SONORAN DESERT
}

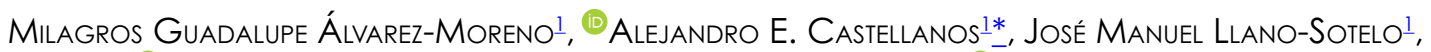 \\ - José Raúl Romo-Leon ${ }^{1}$, Kadiya del Carmen Calderón ${ }^{1}$ y ${ }^{-}$Martín Esqueda $²$
}

\author{
1 DICTUS, Universidad de Sonora. Hermosillo, Sonora, México. \\ ${ }^{2}$ CIAD, A.C., Hermosillo, Sonora, México. \\ *Autor de correspondencia: alejandro.castellanos@unison.mx
}

\section{Resumen}

Antecedentes: Nitrógeno $(\mathrm{N})$ y Fósforo $(\mathrm{P})$ son los nutrientes más limitantes en plantas y su eficiencia de reabsorción sugiere estrategias de conservación. La reabsorción además afecta los cocientes estequiométricos en la hojarasca, modificando las interacciones ecológicas y los ciclos biogeoquímicos en gradientes de aridez.

Hipótesis: Existirá mayor eficiencia de reabsorción de nutrientes en especies de sitios con mayor aridez, por lo que esperamos que los cocientes estequiométricos de C:N y C:P sean mayores en hojas senescentes.

Especies de estudio: Ipomoea arborescens, Fouquieria macdougalii, Cercidium microphyllum, Encelia farinosa, Mimosa laxiflora, Jatropha cardiophylla, Cenchrus ciliaris, Olneya tesota, Prosopis velutina, Cercidium floridum, Fouquieria splendens, Brongniartia minutifolia, Jatropha cordata, Colubrina viridis, Larrea tridentata y Cercidium praecox.

Sitio de estudio y fechas: Tres sitios en un gradiente de aridez del Desierto Sonorense, en la región central de Sonora. Agosto - noviembre 2017 y 2018.

Métodos: Se obtuvieron la eficiencia de reabsorción de N y P, y cocientes estequiométricos C:N, C:P y N:P en hojas verdes y senescentes de especies, comparando tipos funcionales y sitios.

Resultados: La eficiencia de reabsorción disminuyó con el incremento de aridez así como los cocientes estequiométricos C:N y C:P en hojas senescentes y N:P en leguminosas.

Conclusiones: La eficiencia de reabsorción no sugiere una estrategia de conservación de recursos, sin embargo, las diferencias entre tipos funcionales permitieron diferenciar estrategias ecológicas y estequiométricas, en particular las leguminosas, que ayudan a resaltar su influencia en la biogeoquímica de las zonas áridas Sonorenses y posiblemente del país.

Palabras clave: eficiencia de reabsorción, suficiencia de reabsorción, estequiometría ecológica, biogeoquímica, zonas áridas.

\section{Abstract}

Background: Nitrogen $(\mathrm{N})$ and Phosphorus $(\mathrm{P})$ are the most limiting nutrients in plants and their resorption efficiency suggest conservation strategies. Resorption also change litter stoichiometry and affect ecological interactions and biogeochemical cycles along aridity gradients.

Species nutrient resorption efficiency will be higher in sites at the arid extreme, such that resorption proficiency and stoichiometry for C:N and $\mathrm{C}: \mathrm{P}$ will be higher in senescent leaves.

Studied species: Ipomoea arborescens, Fouquieria macdougalii, Cercidium microphyllum, Encelia farinosa, Mimosa laxiflora, Jatropha cardiophylla, Olneya tesota, Prosopis velutina, Cercidium floridum, Fouquieria splendens, Brongniartia minutifolia, Jatropha cordata, Colubrina viridis, Larrea tridentata and Cercidium praecox.

Study site and years of study: Three sites along an aridity gradient in the Sonoran Desert, Central Region in Sonora. August to November 2017 and 2018.

Methods: We obtained N and P reabsorption efficiency, as well as stoichiometric proportions for C:N y N:P from green and senescent leaves to compare species, functional types and sites.

Results: $\mathrm{N}$ and P reabsorption efficiency decreased with aridity and C:N and C:P stoichiometry of senescent leaves, as well as N:P for legumes. Conclusions: Reabsorption efficiency does not suggest a resource conservation strategy, however different functional types allowed us to differentiate ecological and stoichiometric strategies, in particular legumes, that help enhance their role in the biogeochemistry of Sonoran and Mexican arid lands.

Keywords: reabsorption efficiency, resorption proficiency, ecological stoichiometry, biogeochemistry, drylands.

Este artículo se encuentra bajo los términos de la licencia Creative Commons Attribution License CCBY-NC (4.0) internacional. https://creativecommons.org/licenses/by-nc/4.0/ 
Las plantas requieren diferentes elementos químicos para cumplir sus múltiples funciones biológicas y ecológicas (Peñuelas et al. 2019). Algunos se constituyen en nutrientes, de los cuales los más limitantes para la productividad son el nitrógeno $(\mathrm{N})$ y el fósforo $(\mathrm{P})$, pues están relacionados con la síntesis de proteínas, ácidos nucleicos, coenzimas, clorofila, fosfolípidos, moléculas de transferencia de energía, entre otras. La estequiometría ecológica estudia las proporciones entre los elementos y como afectan el funcionamiento biológico, sus interacciones y la biogeoquímica en las comunidades y ecosistemas (Sterner \& Elser 2002). Así, muchos de los atributos de las plantas están relacionados con la concentración de $\mathrm{N}$ y $\mathrm{P}$ foliar, y sus cocientes estequiométricos. Es conocido que las plantas al realizar sus funciones básicas como crecer, fotosintetizar y reproducirse, utilizan los nutrientes más limitantes de manera eficiente para mantener un balance de carbono positivo (Bloom et al. 1985), y así modifican y afectan el tiempo de vida de las hojas, y la calidad de la hojarasca en la senescencia (Sterner \& Elser 2002).

La senescencia es el último proceso de la ontogenia en el que ocurre el desmantelamiento celular, y con la reabsorción (redistribución) de nutrientes desde las hojas hacia otros órganos de la planta, culmina con la muerte celular (Martínez et al. 2008). Con ello podemos entender la eficiencia y la suficiencia de reabsorción; la primera refiere el porcentaje de nutrientes de la hoja que regresa a la planta mientras que la segunda, la concentración de nutrientes que queda en la hoja senescente después de la reabsorción (Killingbeck 1996). La eficiencia de reabsorción se ha relacionado con procesos fisiológicos como la maximización de la fotosíntesis durante el desarrollo foliar en el dosel (Franklin \& Ågren 2002), su longevidad (Eckstein et al. 1999) o con los sumideros de carbono y nutrientes en frutos cercanos (Chapin \& Moilanen 1991). Igualmente, se ha relacionado con la disponibilidad de nutrientes en el suelo (Killingbeck 1996, Côté et al. 2002, Tully et al. 2013, See et al. 2015, Yuan \& Chen 2015) y factores ambientales como precipitación y temperatura (Gerdol et al. 2000, Yuan \& Chen 2009, 2015, Zhao et al. 2017), afectando múltiples funciones biogeoquímicas y del ecosistema (Reed et al. 2012).

Los cocientes estequiométricos C:N y N:P en las hojas pueden o no modificarse por la reabsorción de nitrógeno $(\mathrm{N})$ y fósforo $(\mathrm{P})$ durante la senescencia foliar. Diversos estudios han encontrado eficiencias de reabsorción similares para N y P (Aerts 1996, Vergutz et al. 2012), mientras que otros, rangos muy amplios (Güsewell 2005, Zhao et al. 2017), posiblemente debido a las diferencias en sus reservorios solubles y estructurales (McGroddy et al. 2004). La disimilitud en la estequiometría de C:N y N:P como consecuencia de la reabsorción durante la senescencia puede estar relacionada con funciones de optimización nutrimental para la planta (Chapin \& Kedrowski 1983), y al mismo tiempo con procesos tróficos y biogeoquímicos de los ecosistemas (Mooshammer et al. 2011, Zechmeister-Boltenstern et al. 2015). Sin embargo, ninguno se ha abordado suficientemente.

Un número importante de estudios han mostrado que la proporción $\mathrm{N}: \mathrm{P}$ se incrementa en la hojarasca después de la reabsorción, particularmente bajo condiciones de mayor temperatura y aridez (Wright \& Westoby 2003, Güsewell 2004, See et al. 2015, Prieto \& Querejeta 2020), o en suelos pobres en P (Drenovsky et al. 2019). Las diferencias en la reabsorción entre especies y formas de vida sugieren que la composición de la vegetación y sus cambios, pueden tener efectos importantes en la biogeoquímica de $\mathrm{N}$ y P (Güsewell 2004, Güsewell \& Gessner 2009). Por ejemplo, en el caso de las plantas fijadoras de nitrógeno se ha reportado que suelen reabsorber más $\mathrm{P}$ que $\mathrm{N}$ (Killingbeck 1996, Tateno 2003, Stewart et al. 2008, He et al. 2011), debido a que la fijación del nitrógeno es un proceso energéticamente costoso que requiere fósforo. Por otro lado, el hecho de tener hojas senescentes ricas en nitrógeno hace más fácil su degradación y asimilación por los microorganismos del suelo (Freschet et al. 2013, Mooshammer et al. 2014). Sin embargo, poco se conoce de la estequiometría N:P en la hojarasca y las posibles limitaciones o desbalances de $\mathrm{P}$ después de la reabsorción en especies de zonas áridas y semiáridas.

Aun cuando las zonas áridas son conocidas por una baja disponibilidad y gran heterogeneidad de $\mathrm{N}$ y P en el suelo (Schlesinger et al. 1996, Cross \& Schlesinger 1999, Celaya-Michel et al. 2015), se han encontrado altas concentraciones de nitrógeno foliar en especies del Desierto Sonorense (Castellanos et al. 2018). En algunos estudios se han asociado con su mayor eficiencia de reabsorción en algunos estudios (Campanella \& Bertiller 2011) y menor en otros (Yuan et al. 2005). Es claro que las diferentes correlaciones entre la concentración de $\mathrm{N}$ y P foliares, y su reabsorción sugiere estrategias fisiológicas y ecológicas, así como consecuencias biogeoquímicas contrastantes (Chávez-Vergara et al. 2015), las que son aún poco conocidas en las regiones áridas. Estudios previos en la región árida Sonorense, han mostrado que diferencias en los cocientes estequiométricos de $\mathrm{N}$ y $\mathrm{P}$ están relacionados con el potencial invasivo de las especies (Castellanos et al. 2018), aún cuando previamente se ha considerado el 
agua y no los nutrientes, como el principal factor selectivo en las estrategias ecológicas de plantas desérticas (Austin 2011).

Dado lo anterior, este trabajo contribuye a entender algunos de los procesos que determinan la concentración de $\mathrm{N}$ y $\mathrm{P}$ en tipos funcionales y comunidades del Desierto Sonorense. En particular cómo la eficiencia y suficiencia de reabsorción de N y P modifica la estequiometría foliar durante la senescencia y consecuentemente en la hojarasca. Se plantea que como estrategia de conservación, la eficiencia de reabsorción del nitrógeno será mayor en el extremo más árido, dada su importancia en la fotosíntesis, como se ha sugerido en otros estudios. Como consecuencia, en ese extremo del gradiente, las hojas senescentes tendrán menor concentración de nutrientes y mayores cocientes estequiométricos de C:N y C:P, no así de N:P. Se discuten las estrategias de reabsorción y estequiometría ecológica en diferentes tipos funcionales y leguminosas a la luz de nuestra hipótesis, así como las consecuencias en las dinámicas biogeoquímicas de la hojarasca en las comunidades vegetales del gradiente en donde son dominantes.

\section{Materiales y métodos}

Sitios de estudio. Se seleccionaron tres áreas en la parte central de Sonora, ubicados dentro del Desierto Sonorense. El primero pertenece al municipio de La Colorada, donde se encuentra el Rancho El Churi $(\mathrm{CH})$ ubicado a $28^{\circ} 42^{\prime} 6.77^{\prime}$ ' $\mathrm{N}$ y $-110^{\circ} 32^{\prime} 15.64$ " O, la precipitación media anual (PMA) es $476.0 \mathrm{~mm}$ y la temperatura media anual (TMA) $22.8^{\circ} \mathrm{C}$, el índice de aridez de Martonne (IA $=\mathrm{P} / \mathrm{T}+10$ ) es de 14.51 (árido) y el tipo de vegetación es matorral subtropical y pastizal inducido (Hinojo-Hinojo et al. 2019; Figura 1). El segundo sitio corresponde al Rancho Shangai-Santa Rosalía (SR) ubicado a $29^{\circ} 04^{\prime} 11.94$ " $\mathrm{N}$ y $-110^{\circ} 34^{\prime} 00.78^{\prime \prime} \mathrm{O}$, en el municipio de Hermosillo; la PMA es de $438 \mathrm{~mm}$, la TMA de $24.1^{\circ} \mathrm{C}$ (CONAGUA 2019), con un IA de 12.87 (árido), con vegetación de

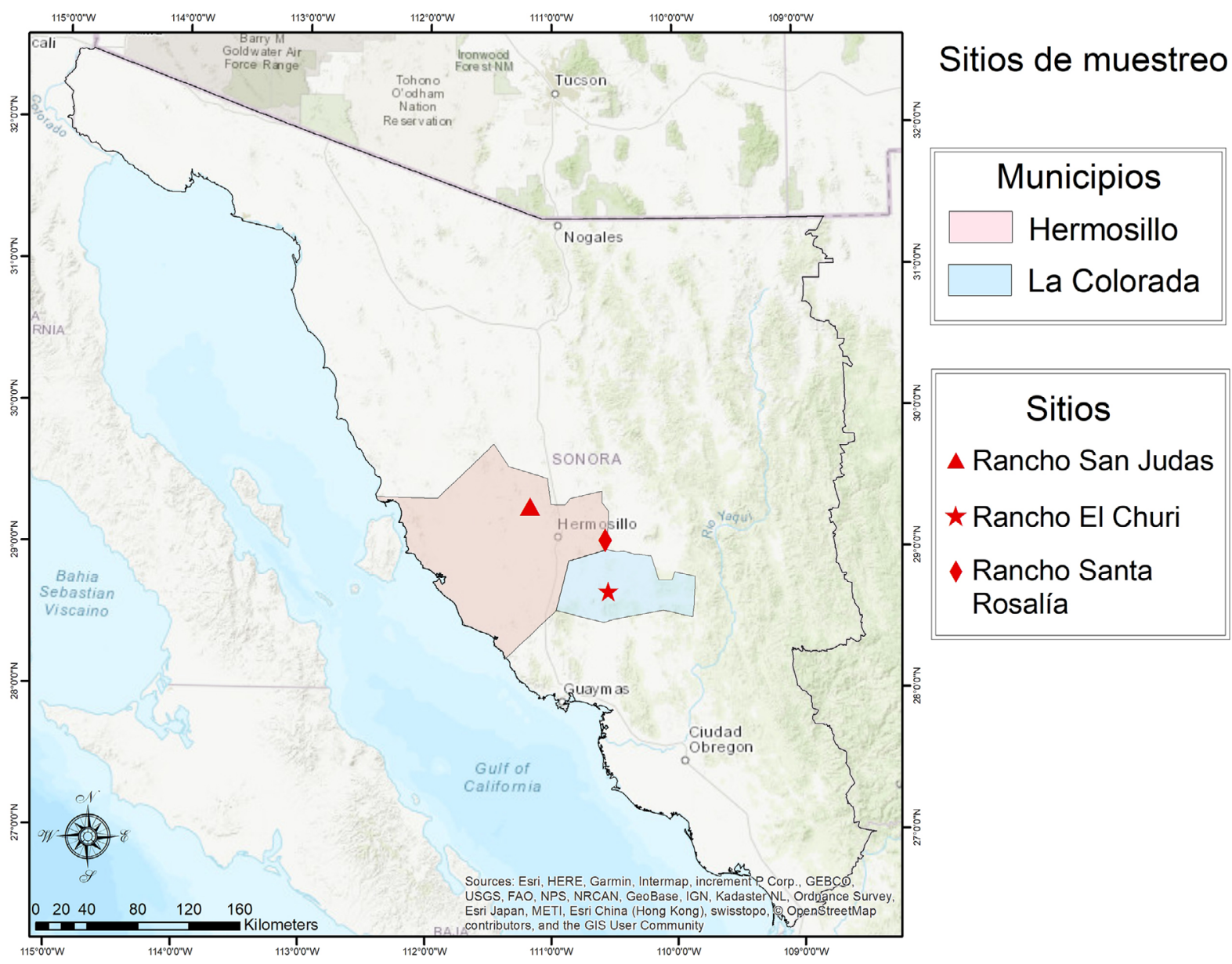

Figura 1. Localización de los sitios de estudio en la región central de Sonora, Noroeste de México. 
mezquital. El tercer sitio, el Rancho San Judas (SJ), se encuentra también en Hermosillo, a $29^{\circ} 17^{\prime} 50.1^{\prime \prime} \mathrm{N} \mathrm{y}-111^{\circ}$ 10' 8.6" O. La PMA es de $385.2 \mathrm{~mm}$, la TMA de $25.1^{\circ} \mathrm{C}$ (CONAGUA 2019), el IA de 10.97 (árido) y vegetación de matorral desértico. Todas las muestras se colectaron entre agosto y noviembre de 2017 y 2018.

Las especies dominantes por sitio de estudio se obtuvieron mediante tres transectos de $150 \mathrm{~m}$ de longitud, utilizando un método al azar estratificado según su forma de vida en árboles y arbustos respectivamente. Posteriormente fueron identificadas y diferenciadas según su tipo funcional basado en sus atributos de perennación foliar (caducifolias o perennifolias) y la facilidad de adquisición de nitrógeno (fijadoras y no fijadoras). En el grupo de las especies fijadoras de nitrógeno se incluyeron también otras leguminosas no fijadoras, dadas sus características funcionales comunes como las altas tasas fotosintéticas y la concentración de nitrógeno foliar reconocidas para todo el taxón (Adams et al. 2016). Por lo tanto, a este grupo funcional lo denominamos como Leguminosas.
Para todas las especies estudiadas se obtuvieron muestras de hojas verdes de cinco individuos (en un par de especies solo cuadruplicado) durante el pico de su desarrollo a mediados de agosto y de sus hojas senescentes en los mismos individuos a finales de septiembre-octubre, para obtener los promedios en cada una de las determinaciones. Con el fin de facilitar las comparaciones de este estudio con otros realizados en la región, todos los nombres científicos siguen los establecidos en Shreve \& Wiggins (1964), aún aquellos con modificaciones taxonómicas recientes (Tabla 1).

Tratamiento de las muestras. En cada uno de los sitios se colectaron hojas verdes maduras y senescentes sin señales de herbivoría o enfermedad. Las hojas verdes provenían de la parte externa del dosel. En el caso de las hojas senescentes, se colocaron trampas de hojarasca, las cuales consistían en mallas de nylon amarradas entre las ramas del individuo, revisadas cada 7 a 15 días (Cornelissen 1996). Las muestras fueron depositadas en sobres de papel rotu-

Tabla 1. Especies dominantes de los sitios de estudio. Las especies fueron diferenciadas por la persistencia de la hoja (caducifolias y perennifolias) y su forma de vida (árbol, arbusto y pasto) así como si son especies de leguminosas (*).

\begin{tabular}{|c|c|c|c|c|}
\hline Sitio & Tipo de hoja & Árbol & Arbusto & Pasto \\
\hline \multirow[t]{5}{*}{ El Churi } & Caducifolia & Ipomoea arborescens & Encelia farinosa & Cenchrus ciliaris \\
\hline & & Fouquieria macdougalii & Mimosa laxiflora* & \\
\hline & & Cercidium microphyllum* & Jatropha cardiophylla & \\
\hline & Perennifolia & Olneya tesota* & & \\
\hline & & Prosopis velutina* & & \\
\hline \multirow[t]{5}{*}{ Santa Rosalía } & Caducifolia & Cercidium microphyllum* & Fouquieria splendens & Cenchrus ciliaris \\
\hline & & Jatropha cordata & Mimosa laxiflora* & \\
\hline & & & Colubrina viridis & \\
\hline & Perennifolia & Olneya tesota* & Brongniartia minutifolia* & \\
\hline & & Prosopis velutina* & & \\
\hline \multirow[t]{4}{*}{ San Judas } & Caducifolia & Cercidium praecox* & Jatropha cardiophylla & \\
\hline & & & Mimosa laxiflora* & \\
\hline & Perennifolia & Olneya tesota* & Larrea tridentata & \\
\hline & & Prosopis velutina* & & \\
\hline
\end{tabular}


ladas (especie, número de individuo, fecha de colecta y tipo de hoja). En hojas senescentes, se limpiaron de posible contaminación (hojas de otras especies, ramas, partes de insectos, etc.) y en ambos casos se transportaron y almacenaron en frío hasta su análisis.

El muestreo de suelo se realizó solo en uno de los sitios de estudio $(\mathrm{CH})$ durante el año 2017. Durante este muestreo se colectó el suelo bajo cinco individuos de las mismas especies arbóreas dominantes con ayuda de una pala, después de retirar la capa superficial de materia orgánica. Las muestras tomadas de los primeros $5 \mathrm{~cm}$ de profundidad se colectaron en bolsas de papel debidamente rotuladas. Las muestras de hojas se secaron en un horno FelisaR FE 243 durante $72 \mathrm{~h}$ a $60{ }^{\circ} \mathrm{C}$ y después se molieron en un molino Wiley; las muestras de suelo se tami- zaron con una malla de $2 \mathrm{~mm}$ y luego secadas de igual forma que las hojas.

Área foliar. El área foliar se midió en hojas verdes y senescentes con un escáner HP Deskjet 2510 y el programa "Image-J" (NIH, EU), colocadas de manera individual junto a una referencia conocida, mantenidas dentro de sobres de papel debidamente identificados, y secadas por $72 \mathrm{~h} \mathrm{a} 60{ }^{\circ} \mathrm{C}$. Después se pesaron individualmente en una balanza analítica y se obtuvo el área foliar específica $(\mathrm{AFE}=$ Área foliar/Peso seco). Se midieron tres hojas por individuo de cada una de las especies.

Nitrógeno y carbono total. Se pesaron $3 \mathrm{mg}$ para hojas y 20 mg para suelo; posteriormente en el analizador elemental

Tabla 2. Media de las concentraciones de N y P para hojas verdes y senescentes de diferentes tipos funcionales en cada sitio. Diferencias estadísticas $(P<=0.05)$ entre tipos funcionales en cada sitio se representan con letras (mayúsculas = diferencias de medias entre sitios; minúsculas $=$ diferencias entre tipos funcionales). NA $=$ No aplica, dada su ausencia en el sitio.

\begin{tabular}{|c|c|c|c|c|c|c|}
\hline & Churi & Sta Rosalía & Sn Judas & Churi & Sta Rosalía & Sn Judas \\
\hline & \multicolumn{3}{|c|}{ Nitrógeno $\left(\mathrm{mgN} \mathrm{g}^{-1}\right)$} & \multicolumn{3}{|c|}{ Fósforo (mgP $\left.\mathbf{g}^{1}\right)$} \\
\hline & \multicolumn{3}{|c|}{ Hojas verdes } & \multicolumn{3}{|c|}{ Hojas verdes } \\
\hline Media & $26.7 \pm 7.1 \mathrm{~B}$ & $26.5 \pm 5.8 \mathrm{~B}$ & $33.6 \pm 4.5 \mathrm{~A}$ & $1.9 \pm 0.7 \mathrm{~B}$ & $1.3 \pm 0.4 \mathrm{C}$ & $2.0 \pm 0.5 \mathrm{~A}$ \\
\hline Arbol & $28.91 \pm 5.7 \mathrm{a}$ & $28.81 \pm 4.4 \mathrm{a}$ & $35.84 \pm 3.9 \mathrm{a}$ & $1.72 \pm 0.5 \mathrm{~b}$ & $1.26 \pm 0.2 \mathrm{~b}$ & $1.64 \pm 0.2 \mathrm{~b}$ \\
\hline Arbusto & $27.46 \pm 5.6 \mathrm{a}$ & $25.6 \pm 6.5 \mathrm{ab}$ & $31.58 \pm 4.0 \mathrm{~b}$ & $1.78 \pm 0.6 \mathrm{~b}$ & $1.13 \pm 0.2 \mathrm{c}$ & $2.28 \pm 0.6 \mathrm{a}$ \\
\hline Pasto & $13.68 \pm 0.6 b$ & $21.22 \pm 3.9 b$ & NA & $3.38 \pm 0.4 \mathrm{a}$ & $2.29 \pm 0.6 \mathrm{a}$ & NA \\
\hline Caducifolia & $24.91 \pm 6.8 \mathrm{~b}$ & $24.09 \pm 5.2 \mathrm{~b}$ & $35.31 \pm 4.4 \mathrm{a}$ & $2.03 \pm 0.8 \mathrm{~b}$ & $1.31 \pm 0.5 \mathrm{c}$ & $2.23 \pm 0.6 \mathrm{a}$ \\
\hline Perennifolia & $32.73 \pm 4.1 \mathrm{a}$ & $31.43 \pm 3.6 \mathrm{a}$ & $31.53 \pm 3.7 \mathrm{a}$ & $1.58 \pm 0.3 \mathrm{~b}$ & $1.33 \pm 0.2 \mathrm{c}$ & $1.70 \pm 0.2 \mathrm{a}$ \\
\hline Leguminosa & $31.26 \pm 4.3 \mathrm{~b}$ & $29.67 \pm 4.6 b$ & $34.38 \pm 4.3 \mathrm{a}$ & $1.61 \pm 0.5 \mathrm{~b}$ & $1.26 \pm 0.2 \mathrm{c}$ & $1.80 \pm 0.3 \mathrm{a}$ \\
\hline \multirow[t]{2}{*}{ Otras } & $25.30 \pm 5.0 \mathrm{~b}$ & $23.1 \pm 5.1 \mathrm{~b}$ & $32.07 \pm 4.6 \mathrm{a}$ & $1.82 \pm 0.5 \mathrm{~b}$ & $1.08 \pm 0.2 \mathrm{c}$ & $2.33 \pm 0.7 \mathrm{a}$ \\
\hline & \multicolumn{3}{|c|}{ Hojas senescentes } & \multicolumn{3}{|c|}{ Hojas senescentes } \\
\hline Media & $12.8 \pm 8.7 \mathrm{~B}$ & $13.4 \pm 7.6 \mathrm{~B}$ & $21.8 \pm 8.1 \mathrm{~A}$ & $1.0 \pm 0.4 \mathrm{~B}$ & $0.6 \pm 0.3 \mathrm{C}$ & $1.5 \pm 0.7 \mathrm{~A}$ \\
\hline Árbol & $15.78 \pm 10.42 \mathrm{a}$ & $17.48 \pm 8.33 \mathrm{a}$ & $28.38 \pm 6.53 \mathrm{a}$ & $1.10 \pm 0.48 \mathrm{a}$ & $0.82 \pm 0.39 \mathrm{a}$ & $1.54 \pm 0.54 \mathrm{a}$ \\
\hline Arbusto & $11.18 \pm 3.59 \mathrm{a}$ & $11.63 \pm 4.45 \mathrm{~b}$ & $16.15 \pm 3.78 b$ & $0.97 \pm 0.37 \mathrm{ab}$ & $0.54 \pm 0.15 b$ & $1.54 \pm 0.81 \mathrm{a}$ \\
\hline Pasto & $4.4 \pm 0.65 \quad b$ & $4.3 \pm 2.16 \mathrm{c}$ & NA & $0.63 \pm 0.09 \mathrm{c}$ & $0.39 \pm 0.08 \mathrm{c}$ & NA \\
\hline Caducifolia & $9.16 \pm 3.9 \mathrm{~b}$ & $10.02 \pm 6.0 \mathrm{~b}$ & $19.2 \pm 6.3 \mathrm{a}$ & $0.88 \pm 0.31 \mathrm{~b}$ & $0.54 \pm 0.18 \mathrm{c}$ & $1.74 \pm 0.7 \mathrm{a}$ \\
\hline Perennifolia & $26.82 \pm 7.6 \mathrm{a}$ & $20.2 \pm 5.5 \mathrm{~b}$ & $24.86 \pm 9.1 \mathrm{~b}$ & $1.46 \pm 0.52 \mathrm{a}$ & $0.87 \pm 0.42 \mathrm{~b}$ & $1.32 \pm 0.7 \mathrm{ab}$ \\
\hline Leguminosa & $20.07 \pm 9.2 b$ & $18.33 \pm 6.2 b$ & $25.62 \pm 7.5 \mathrm{a}$ & $1.31 \pm 0.5 \mathrm{~b}$ & $0.80 \pm 0.4 \mathrm{c}$ & $1.79 \pm 0.7 \mathrm{a}$ \\
\hline Otras & $8.45 \pm 1.7 \mathrm{~b}$ & $8.26 \pm 3.4 b$ & $15.00 \pm 2.9 \mathrm{a}$ & $0.83 \pm 0.2 \mathrm{a}$ & $0.47 \pm 0.1 \mathrm{~b}$ & $1.10 \pm 0.4 \mathrm{a}$ \\
\hline
\end{tabular}



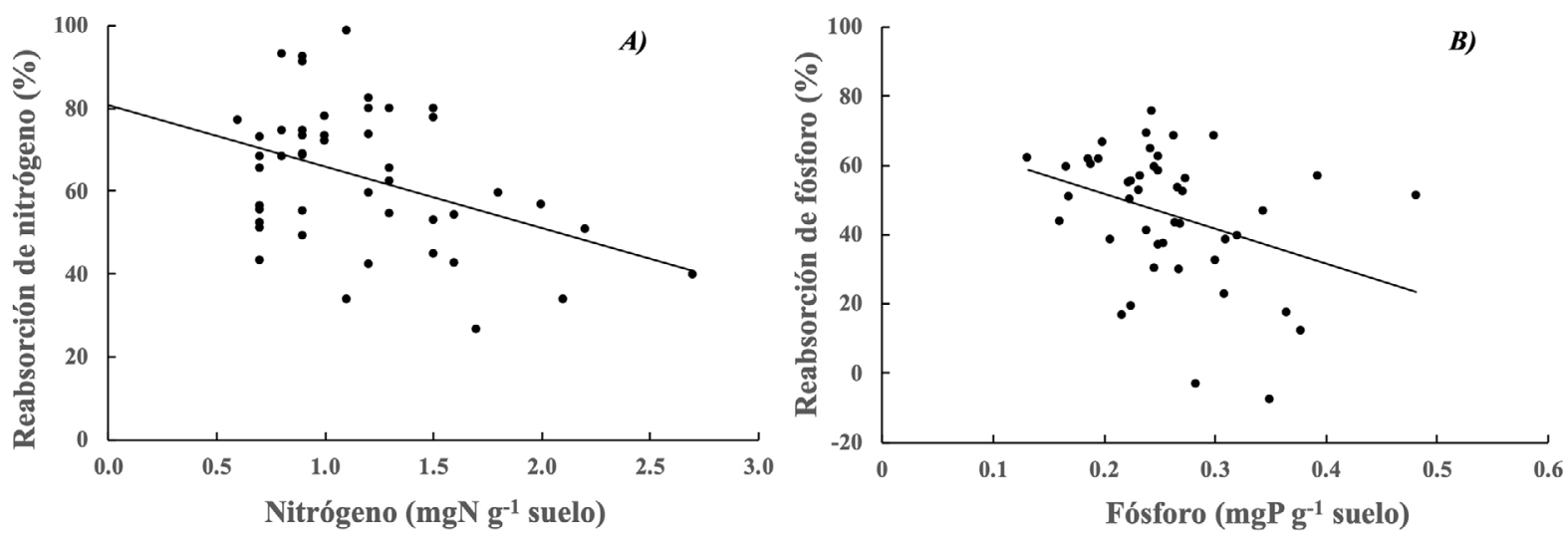

Figura 2. Relación de la eficiencia de reabsorción de A) nitrógeno (ERN) y B) fósforo (ERP) en hojas de diferentes especies y la concentración de $\mathrm{N}$ o $P$ en el suelo $\left(E R N=-4.836 \times \mathrm{N}+80.826 ; R^{2}=0.17\right.$ y ERP $\left.=-101.16 * P+71.881 ; R^{2}=0.12\right)$. Datos son para el sitio menos árido del gradiente de estudio $(\mathrm{CH})$ en 2017.

PerkinElmer 2400 serie II mediante la combustión completa de la muestra $\left(950-1300{ }^{\circ} \mathrm{C}\right.$ y atmósfera de oxígeno puro) para convertir los elementos en gases simples (C y $\mathrm{N})$, separarlos y medirlos, para obtener la concentración de cada elemento en la muestra.

Fósforo total. Para hojas se requirió de $150 \mathrm{mg}$ y $10 \mathrm{~g}$ para suelo. Las muestras secas fueron digeridas en una mezcla de ácido sulfúrico y sulfato de potasio, mediante la reacción con molibdato de amonio y ácido ascórbico, y después medido a $660 \mathrm{~nm}$ en el analizador AA1 (SEAL) rápido de fluidos (Murphy \& Riley 1962).

Eficiencia de reabsorción. Se determinó la reabsorción de nutrientes (N y P) mediante la fórmula (Van Heerwaarden et al. 2003):

Reabsorción de Nutrientes $(\%)=\left[\left(1-\left(\mathrm{Nu}_{\mathrm{sen}} / \mathrm{Nu}_{\mathrm{ver}}\right) \times(\mathrm{FCPM})\right] \times 100\right.$

donde $\mathrm{Nu}_{\text {sen }}$ y $\mathrm{Nu}_{\text {ver }}$ son las concentraciones de nutrientes en la hoja senescente y la hoja verde respectivamente. Los valores de reabsorción de nutrientes consideraron la corrección por pérdida de masa (FCPM), que puede inducir errores en el cálculo ante un cambio significativo de masa foliar en la senescencia (Vergutz et al. 2012). Sin embargo, otros estudios recientes no consideran dicho factor de corrección (See et al. 2015, Sohrt et al. 2018), por lo que es importante señalarlo para comparar los resultados con la mayor cantidad de estudios recientes.

Los cocientes estequiométricos se obtuvieron a partir de las concentraciones de nutrientes foliares totales, con los valores obtenidos en base a peso ( $\mathrm{g}$ Nutriente $\mathrm{g}^{-1}$ hoja) de cada individuo por especie estudiada. Las relaciones de $\mathrm{C}: \mathrm{N}, \mathrm{N}: \mathrm{P}$ y $\mathrm{C}: \mathrm{P}$ se determinaron tanto en hojas verdes como senescentes.

Análisis estadístico. Las especies estudiadas se agruparon por sitio, los que se compararon a lo largo del gradiente para conocer las diferencias entre las concentraciones foliares promedio, sus cocientes estequiométricos en las hojas maduras verdes y las senescentes, así como su eficiencia y suficiencia de reabsorción. Los análisis estadísticos se realizaron utilizando el programa JMP v14.0.0 de SAS. Se realizaron pruebas de normalidad a los datos, sin que algunos pudieran ajustarse aún después de ser transformados con funciones logarítmicas, por lo que los datos se analizaron mediante pruebas no paramétricas y contrastadas con un intervalo de confianza al $95 \%$.

Se realizaron ANOVAs con la prueba de Wilcoxon / Kruskal-Wallis para muestras independientes y de Wilcoxon para mediciones repetidas, al comparar las concentraciones foliares de nutrientes, su eficiencia y suficiencia de reabsorción, los cocientes estequiométricos en hojas verdes y senescentes entre sitios, y luego entre los tipos funcionales. Para las correlaciones en el suelo en cuanto a la eficiencia de reabsorción de $\mathrm{N}$ y $\mathrm{P}$ con su concentración total en el suelo, se realizó una regresión lineal simple, con la estimación M de Huber de ajuste robusto.

\section{Resultados}

Relación de las concentraciones de nutrientes entre las hojas y el suelo. Se compararon las eficiencias de reabsorción de nutrientes foliares ( $\mathrm{N}$ y $\mathrm{P}$ ) de todos los individuos de las especies dominantes estudiadas con la concentración 
del nutriente en el suelo para el sitio $\mathrm{CH}$ y se encontró una relación inversa entre la eficiencia de reabsorción de nitrógeno $\left(F_{(1,44)}=8.75, P=0.005, R^{2}=0.17\right) \mathrm{y}$ fósforo $\left(F_{(1,41)}=6.11, P=0.017, R^{2}=0.12\right)$ de las hojas con la concentración total del mismo nutriente en el suelo (Figura 2).

Nutrientes en hojas verdes y senescentes. Se encontraron diferencias significativas en las concentraciones de nitrógeno foliar entre las hojas verdes y senescentes entre los sitios. En todos los casos las mayores concentraciones de nitrógeno $(\mathrm{N})$ y de fósforo $(\mathrm{P})$ correspondientes a San Judas (SJ), el sitio más árido en el estudio (Tabla 2). Por otro lado, la concentración de nitrógeno en hojas de árboles fue mayor que las de otras formas de vida en los tres sitios, tanto en hojas verdes como senescentes, aunque solo en San Judas las diferencias fueron significativas con arbustos (Tabla 2). En contraste, las concentraciones de $\mathrm{P}$ en hojas verdes fueron mayores en herbáceas y pastos, y menores en arbustos y árboles respectivamente. Sin embargo, los arbustos en SJ presentaron las concentraciones más altas en hoja verde (Tabla 2).

Según sus atributos funcionales, las concentraciones promedio de $\mathrm{N}$ en hojas verdes fueron mayores para especies perennifolias que caducifolias en los sitios $\mathrm{CH}$ y $\mathrm{SR}$, pero no en el sitio más árido de SJ (Tabla 2). En contraste, la concentración de $\mathrm{P}$ en hojas verdes fue mayor en especies caducifolias de ambos extremos del gradiente, $\mathrm{CH}$ y SJ. Además, en SJ se encontraron las mayores concentraciones de $\mathrm{P}$ en hojas senescentes de especies caducifolias, es decir menor suficiencia, mientras que en los otros sitios, el $\mathrm{P}$ fue mayor en especies perennifolias (Tabla 2).

Las leguminosas definidas como tipo funcional (ver método) tuvieron mayor concentración de nitrógeno foliar (N) en hojas verdes y senescentes que las otras especies. Las concentraciones de $\mathrm{N}$ más altas en hojas verdes se presentaron en SJ, lo mismo que en las hojas senescentes, al igual que para las otras especies (Tabla 2). Las concentraciones foliares de $\mathrm{P}$ en hojas verdes y senescentes fueron menores en el sitio intermedio (SR) comparado con ambos extremos del gradiente, $\mathrm{CH}$ y $\mathrm{SJ}$, en donde una diferencia importante fue que las leguminosas tuvieron significativamente menor concentración de $\mathrm{P}$ en hojas verdes (Tabla 2). De manera importante encontramos que las mayores concentraciones de $\mathrm{P}$ en hojas senescentes (menor suficiencia) correspondieron al tipo funcional de leguminosas en todos los sitios (Tabla 2).

Eficiencia de reabsorción. No se observaron diferencias significativas entre los promedios de las eficiencias de re-
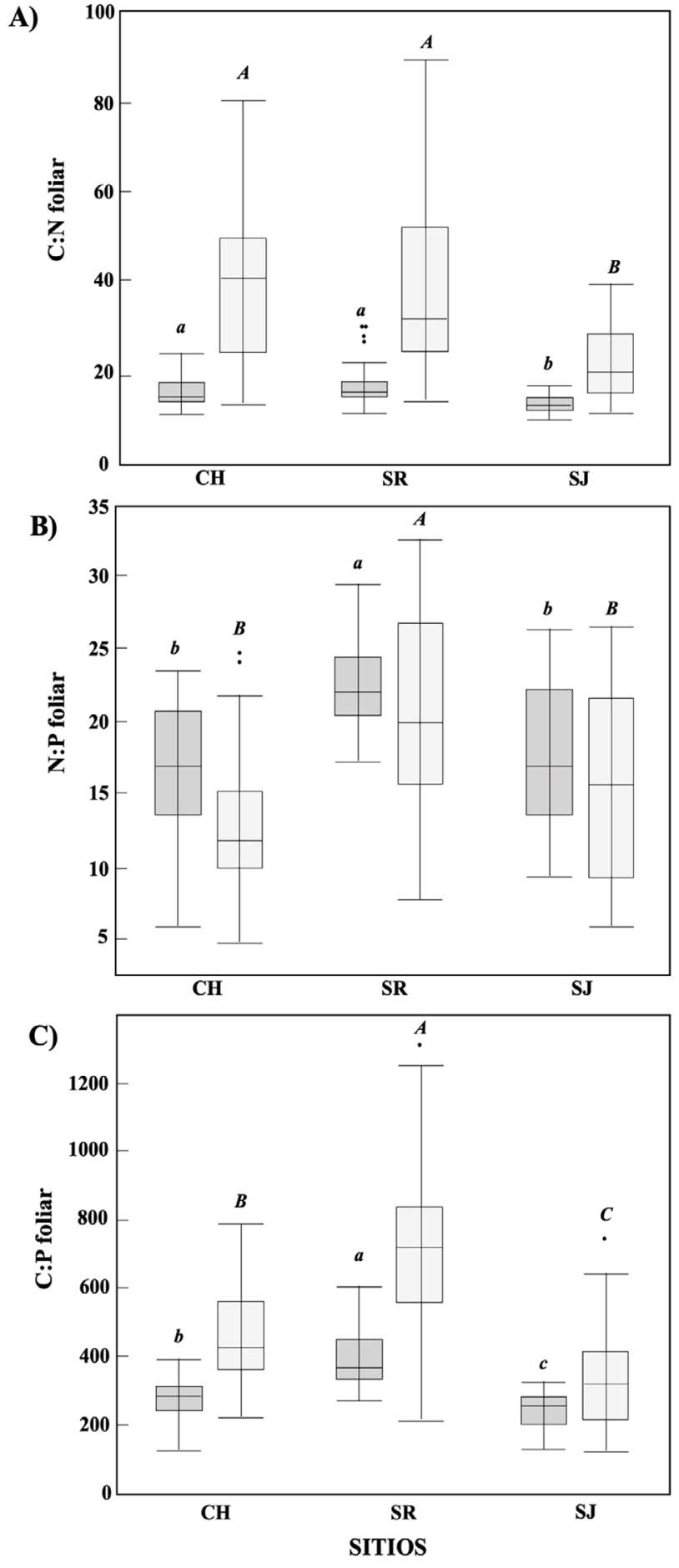

Figura 3. Gráficas de cajas mostrando los cuartiles y medianas de los cocientes estequiométricos de A) C:N, B) N:P y C) C:P en hojas verdes (gris oscuro) y senescentes (gris claro) para cada sitio de estudio. Letras muestran diferencias significativamente mayores a $P=0.005$ entre sitios para hojas verdes (minúsculas) y senescentes (mayúsculas). 
Reabsorción de N y P

Tabla 3. Eficiencia (\%) y suficiencia (mgNutriente $\left.\mathrm{g}^{-1}\right)$ de reabsorción en tres sitios en un gradiente de menor $(\mathrm{CH})$ a mayor $(\mathrm{SJ})$ aridez. Valores representan la media \pm d.s. Diferencias estadísticas $(P<=0.05)$ se señalan con letras.

\begin{tabular}{ccccc}
\hline Sitio & \multicolumn{2}{c}{ Eficiencia de reabsorción } & \multicolumn{2}{c}{ Suficiencia de reabsorción } \\
\hline & $\mathbf{N}$ & $\mathbf{P}$ & $\mathbf{N}$ & $\mathbf{P}$ \\
\hline El Churi & $53.6 \pm 22.8 \mathrm{a}$ & $42.2 \pm 30.7 \mathrm{a}$ & $12.8 \pm 8.7 \mathrm{~b}$ & $1.0 \pm 0.4 \mathrm{~b}$ \\
Santa Rosalía & $51.7 \pm 20.7 \mathrm{a}$ & $48.7 \pm 23.9 \mathrm{a}$ & $13.4 \pm 7.6 \mathrm{~b}$ & $0.64 \pm 0.3 \mathrm{c}$ \\
San Judas & $35.1 \pm 20.8 \mathrm{~b}$ & $20.4 \pm 33.9 \mathrm{~b}$ & $21.8 \pm 8.1 \mathrm{a}$ & $1.5 \pm 0.7 \mathrm{a}$ \\
\hline
\end{tabular}

absorción de nitrógeno de las especies muestreadas en los sitios menos áridos $(\mathrm{SR}=51.7 \pm 0.7 \% \mathrm{~N}$ y $\mathrm{CH}=53.6$ $\pm 22.8 \% \mathrm{~N}$ ), y solo fueron significativas al compararlas con el sitio más árido $\left(\mathrm{SJ}, \chi_{(2,102)}^{2}=10.21 ; P=0.006\right)$ dada la menor reabsorción en SJ $(35.1 \pm 20.8 \% \mathrm{~N})$. Las diferencias en la reabsorción de fósforo apenas fueron significativas entre sitios $\left(\chi_{(2,100)}^{2}=8.62, P=0.01\right)$, por las diferencias entre el sitio con especies que presentaron el mayor promedio en su eficiencia de reabsorción del nutriente $(\mathrm{SR}=48.7 \pm 23.9 \%)$ y el sitio con menor promedio en sus especies $(\mathrm{SJ}=20.4 \pm 33.9 \%$; Tabla 3 ).

Las diferencias en la reabsorción de $\mathrm{N}$ y $\mathrm{P}$ entre sitios estuvieron fuertemente influenciadas por las tasas de reabsorción específicas. Hubo tendencias importantes entre las especies que conforman las comunidades vegetales en los tres sitios que fueron determinantes en la gran variabilidad encontrada. Por ejemplo, en SJ la diferencia entre la mayor ( $\mathrm{J}$. cardiophylla $=64.7 \pm 8.1)$ y menor $(O$. tesota $=8.76 \pm 17.7)$ eficiencia de reabsorción fue de casi un orden de magnitud (Tabla 4). En el extremo menos árido $(\mathrm{CH})$ las diferencias en la eficiencia de reabsorción de nitrógeno (ERN) fueron también significativas entre las especies $\left(\chi_{(7,34)}^{2}=22.28, P<0.002\right)$, pero la variación en la eficiencia entre especies fue menor (solo cinco veces entre el valor más alto en $I$. arborecens y el menor en $O$. tesota). Esta variación fue similar a la encontrada en las especies en el sitio intermedio (SR), en donde la mayor ERN ( $J$. cordata y $F$. splendens) fue casi cuatro veces la menor $(P$. velutina; Tabla 4).

De la misma manera, las variaciones en la eficiencia de reabsorción de fósforo (ERP) fueron aún mayores entre las especies de cada sitio; algunas con un aumento en la concentración del nutriente durante la senescencia y como consecuencia valores negativos. La mayoría de las especies que incrementaron su concentración durante la senescencia se ubicaron en el sitio de mayor aridez (SJ), destacando $M$. laxiflora en SJ como la especie con mayor incremento del nutriente en sus hojas senescentes, al igual que otras especies de leguminosas como $P$. velutina $(\mathrm{SJ}) \mathrm{y}$ O. tesota (en SJ y SR) (Tabla 4).

Cocientes estequiométricos. Los cocientes $\mathrm{C}: \mathrm{N}, \mathrm{N}: \mathrm{P}$ y $\mathrm{C}: \mathrm{P}$ foliares fueron analizados entre los diferentes sitios del gradiente de aridez. El cociente $\mathrm{C}: \mathrm{N}$ en hoja verde $\left(\chi_{(2,103)}^{2}=20.86, P<0.0001\right)$ y senescente $\left(\chi_{(2,103)}^{2}=19.94\right.$, $P<0.0001)$ mostró diferencias entre los sitios, pero en ambos casos fue significativamente menor en el sitio más árido $(\mathrm{SJ}=13.59 \pm 1.99$ y $22.97 \pm 7.7$ en hojas verdes y senescentes respectivamente) (Figura 3a). En cambio, las diferencias en el cociente $\mathrm{N}: \mathrm{P}$ en hojas verdes $\left(\chi_{(2,103)}^{2}\right.$ $=32.84, P<0.0001)$ y senescentes $\left(\chi_{(2,101)}^{2}=26.42, P<\right.$ $0.0001)$, mostraron mayor variabilidad y solo fueron diferentes entre el sitio intermedio, pero no entre los extremos del gradiente (Figura $3 \mathrm{~b})$. El cociente C:P mostró también gran variabilidad (Figura 3c), aunque las diferencias entre los sitios fueron estadísticamente significativas. Los cocientes estequiométricos $\mathrm{C}: \mathrm{N}$ y $\mathrm{C}: \mathrm{P}$ aumentaron en hojas senescentes, mientras que $\mathrm{N}: \mathrm{P}$ disminuyeron.

\section{Discusión}

En este estudio se buscó caracterizar las estrategias de conservación de recursos, mediante la eficiencia de reabsorción de nutrientes, entre los diferentes grupos funcionales de las especies vegetales dominantes en tres comunidades del Desierto Sonorense. El análisis de las concentraciones de $\mathrm{N}$ y $\mathrm{P}$, su eficiencia y suficiencia de reabsorción, y las relaciones estequiométricas durante la senescencia de las hojas en diferentes grupos funcionales, se utilizó para entender algunas de las características que permiten prefigurar su nicho biogeoquímico (sensu Peñuelas et al. 2008, 2019). Nuestro estudio aporta mayores elementos al esclarecimiento de los procesos estequiométricos y ecológicos que afectan las relaciones biogeoquímicas e 
Álvarez-Moreno et al. / Botanical Sciences 99(3): 499-513. 2021

Tabla 4. Eficiencia de reabsorción (\%) considerando el factor de corrección de las especies estudiadas en cada uno de los sitios. Valores representan la media \pm d.s y letras las diferencias estadísticas. \# denota que la especie no fué incluida en los análisis estadísticos.

\begin{tabular}{|c|c|c|c|}
\hline Especie & San Judas & Santa Rosalía & El Churi \\
\hline \multicolumn{4}{|c|}{ Eficiencia de reabsorción de nitrógeno } \\
\hline B. minutifolia & - & $56.64 \pm 14.29 \mathrm{ab}$ & - \\
\hline C. ciliaris & - & $87.29 \pm 5.26^{\#}$ & $71.23 \pm 3.01$ \# \\
\hline C. microphyllum & - & $48.54 \pm 24.74 \mathrm{abc}$ & $58.65 \pm 16.04 \mathrm{ab}$ \\
\hline C. praecox & $36.41 \pm 15.02 \mathrm{abc}$ & - & - \\
\hline C. viridis & - & $57.74 \pm 11.32 \mathrm{ab}$ & - \\
\hline E. farinosa & - & - & $67.37 \pm 2.94 \mathrm{ab}$ \\
\hline F. macdougalii & - & - & $67.23 \pm 7.64 \mathrm{ab}$ \\
\hline F. splendens & - & $69.28 \pm 12.80 \mathrm{a}$ & - \\
\hline I. arborescens & - & - & $78.59 \pm 3.56 \mathrm{a}$ \\
\hline J. cordata & - & $78.04 \pm 2.83 \mathrm{a}$ & - \\
\hline J. cardiophylla & $64.72 \pm 11.6 \mathrm{a}$ & - & $43.86 \pm 20.7 \mathbf{b c}$ \\
\hline L. tridentata & $61.65 \pm 11.71 \mathrm{ab}$ & - & - \\
\hline M. laxiflora & $31.48 \pm 17.81 \mathrm{bc}$ & $58.88 \pm 13.62 \mathrm{ab}$ & $56.62 \pm 17.45 \mathrm{ab}$ \\
\hline O. tesota & $8.76 \pm 17.7 \mathrm{c}$ & $27.7 \pm 14.6 \mathrm{bc}$ & $13.74 \pm 9.79 \mathrm{c}$ \\
\hline P. velutina & $11.58 \pm 15.5 \mathrm{c}$ & $14.75 \pm 30.5 \mathrm{c}$ & $36.30 \pm 27.13 \mathrm{bc}$ \\
\hline \multicolumn{4}{|c|}{ Eficiencia de reabsorción de fósforo } \\
\hline B. minutifolia & - & $58.2 \pm 13.4 \mathrm{ab}$ & - \\
\hline C. ciliaris & - & $88.52 \pm 3.70^{\#}$ & $83.11 \pm 2.20^{\#}$ \\
\hline C. microphyllum & - & $43.02 \pm 26.4 \mathrm{abc}$ & $31.29 \pm 26.26 \mathrm{ab}$ \\
\hline C. praecox & $20.83 \pm 19.17 \mathrm{bc}$ & - & - \\
\hline C. viridis & - & $54.85 \pm 13.1 \mathrm{abc}$ & - \\
\hline E. farinosa & - & - & $53.47 \pm 8.18 \mathrm{a}$ \\
\hline F. macdougalii & - & - & $56.43 \pm 11.57 \mathrm{a}$ \\
\hline F. splendens & - & $58.71 \pm 15.9 \mathrm{ab}$ & - \\
\hline I. arborescens & - & - & $65.22 \pm 5.04 \mathrm{a}$ \\
\hline J. cardiophylla & $50.90 \pm 9.63 \mathrm{ab}$ & - & $38.69 \pm 14.51 \mathrm{ab}$ \\
\hline J. cordata & - & $67.05 \pm 2.9 \mathrm{a}$ & - \\
\hline L. tridentata & $73.69 \pm 6.9 \mathrm{a}$ & - & - \\
\hline M. laxiflora & $-25.28 \pm 19.29 d$ & $56.56 \pm 13.2 \mathrm{abc}$ & $33.75 \pm 27.12 \mathrm{ab}$ \\
\hline O. tesota & $-1.99 \pm 25.63 \mathrm{~cd}$ & $-2.9 \pm 37.38 \mathrm{c}$ & $2.19 \pm 6.55 b$ \\
\hline P. velutina & $-7.21 \pm 16.5 \mathrm{~cd}$ & $3.7 \pm 61.3 \mathrm{bc}$ & $22.87 \pm 36.50 \mathrm{ab}$ \\
\hline
\end{tabular}


interacciones ecológicas por diferentes grupos funcionales en el Desierto Sonorense.

Concentraciones foliares en el gradiente de aridez. En el extremo más árido del gradiente (SJ), se encontraron las concentraciones más altas para $\mathrm{N}$ foliar de los tres sitios estudiados. Diversos autores correlacionan de manera positiva la concentración de $\mathrm{N}$ en las hojas y la aridez (West \& Skujins 1978, Wright \& Westoby 2002). De mayor a menor aridez, las concentraciones foliares de nitrógeno en hojas verdes fueron decreciendo y aumentando en su variabilidad. Las concentraciones foliares que se han reportado en metaanálisis globales, incluyendo 753 especies a lo largo de China, son cercanas a $18.4 \pm 0.5 \mathrm{mgN} \mathrm{g}^{-1}$ (Han et al. 2005, Vergutz et al. 2012), lo que significan valores menores que los promedios encontrados para los tres sitios en este estudio. Al igual que en hojas verdes, también en senescentes encontramos una mayor concentración de $\mathrm{N}$ que el promedio global de $9.74 \pm 0.33 \mathrm{mgN} \mathrm{g}^{-1}$ ( al. 2005, Vergutz et al. 2012), aún en el sitio menos árido que promedió la menor concentración $\left(12.8 \pm 8.7 \mathrm{mgN} \mathrm{g}^{-1}\right.$ en $\mathrm{CH}$ ).

Los patrones de concentración fueron diferentes con el P. En hojas verdes la mayor concentración promedio ocurrió en ambos extremos del gradiente, que resultaron mayores al promedio global de $1.4 \pm 0.01 \mathrm{mgP} \mathrm{g}^{-1}$ ( al. 2005, Kobe et al. 2005, Vergutz et al. 2012). Una alta concentración de $\mathrm{P}$ se ha relacionado con hojas de rápido crecimiento y caducifolias (Drenovsky et al. 2019), tal como se presentó en el gradiente estudiado (Tabla 2). De manera similar, el $\mathrm{P}$ en hojas senescentes fue mayor en el extremo más árido $\left(1.5 \pm 0.7 \mathrm{mgP} \mathrm{g}^{-1} \mathrm{en} \mathrm{SJ}\right)$, también más alto al promedio global de $0.8 \pm 0.01 \mathrm{mgP} \mathrm{g}^{-1}$ reportado previamente (Han et al. 2005, Kobe et al. 2005, Vergutz et al. 2012).

El hecho de que las concentraciones foliares sean altas en los grupos funcionales en un sitio puede deberse a una alta concentración inicial por diversos mecanismos de asignación de recursos, incluida una mayor eficiencia de reabsorción de nutrientes, mayor disponibilidad del nutriente en el suelo, o a mecanismos de incorporación que faciliten su absorción como puede ser el establecimiento con asociaciones simbióticas y/o micorrícicas (Bonfante \& Genre 2010). Sin embargo, una mayor concentración de P en hojas senescentes, puede no resultar en mayor aprovechamiento por las comunidades microbianas o las mismas plantas, dadas las condiciones impredecibles de la precipitación. La estrategia de estas especies ante las condiciones ambientales en las que se desarrollan debe ser estudiada con mayor detalle, para comprender mejor los mecanismos que controlan las interacciones entre la concentración de nutrientes en la hojarasca, la eficiencia de reabsorción y las estrategias biogeoquímicas e interacciones ecológicas con los microorganismos en el suelo (Brant \& Chen 2015).

Eficiencia de reabsorción. Se observó una disminución en la eficiencia de reabsorción de $\mathrm{N}$ y $\mathrm{P}$ en promedio para las especies en sitios con condiciones de mayor aridez. En lo que respecta a $\mathrm{N}$, la menor eficiencia de reabsorción en condiciones de mayor aridez fue significativamente diferente $(\mathrm{SJ}=35.1 \pm 20.8 \%$; Tabla 3 ). De manera similar, la eficiencia de reabsorción de $\mathrm{P}$ fue menor en promedio en especies del sitio con mayor aridez ( $\mathrm{SJ}=20.4 \pm 33.9 \%$; Tabla 3). Se ha reportado menor eficiencia de reabsorción de $\mathrm{N}$ con la aridez, mientras que la concentración en la hoja senescente aumenta (Bertiller et al. 2005), acorde a lo encontrado en este estudio (Tabla 3). Sin embargo, eso contrasta con las diferencias obtenidas en la eficiencia de reabsorción en otros estudios. Por ejemplo, especies de clima mediterráneo con diferentes ecotipos no mostraron cambios en la eficiencia de reabsorción, aun cuando los reservorios de nutrientes fueron diferentes entre sitios (Pugnaire \& Chapin 1993), lo mismo que encontró $\underline{\text { Aerts }}$ (1996) incluso en perennifolias, sugiriendo que la eficiencia de reabsorción no representa una estrategia de conservación de recursos en las especies estudiadas. Lo anterior también coincide con este trabajo, dado que encontramos diferencias en la eficiencia de reabsorción para las mismas especies entre los sitios (Tabla 4).

La eficiencia de reabsorción de $\mathrm{N}$ y $\mathrm{P}$ se modifica en las especies y consecuentemente en los sitios a lo largo de un gradiente de aridez. Sin embargo, nuestros datos coinciden en que no hubo una tendencia hacia la mayor conservación de nutrientes en sitios de mayor aridez, dada la menor eficiencia de reabsorción de N y P. Es posible, que la menor eficiencia de reabsorción promedio de las hojas en el sitio de mayor aridez, se compense por su mayor concentración foliar inicial, lo que significa que la cantidad de nutriente reabsorbido (al menos en el caso del N), sería muy similar entre los sitios del gradiente, aun cuando los nutrientes que llegan al suelo a través de la hojarasca fueran disímiles.

Es claro que la eficiencia de reabsorción de nutrientes tiene implicaciones directas sobre la disponibilidad de nutrientes y la fisiología del individuo en las especies (Aerts 1996), mientras que la suficiencia y estequiometría de la reabsorción tendrá impactos en la tasa de descomposición del suelo (Killingbeck 1996, Chávez-Vergara et al. 2018) 
y los ciclos biogeoquímicos. Las especies más suficientes son aquellas que retienen las menores cantidades de nutrientes en sus hojas senescentes, comúnmente iguales o menores a $7 \mathrm{mgN} \mathrm{g}^{-1}$ para $\mathrm{N}$ y $0.5 \mathrm{mgP} \mathrm{g}^{-1}$ para $\mathrm{P}$ (Killingbeck 1996). Las altas concentraciones de nutrientes en hojas senescentes encontradas en este estudio, particularmente en las leguminosas que incluyen tanto fijadoras como no fijadoras (Tabla 2), sugieren la posibilidad de una reabsorción incompleta y consecuentemente una mayor concentración y disponibilidad regresando al suelo (Schlesinger \& Pilmanis 1998). Una disponibilidad de nutrientes más alta en el suelo puede beneficiar a microorganismos, bacterias y/o micorrizas, que a su vez pueden incrementar las formas disponibles de $\mathrm{N}$ y $\mathrm{P}$ para las plantas en la comunidad (Bonfante \& Genre 2010, Adams et al. 2016). Una característica relevante en el área de estudio y en general de la región más subtropical del Desierto Sonorense (Shreve \& Wiggins 1964, Turner et al. 1995, Castellanos et al. 2010) es la abundancia de especies de leguminosas, lo que sugiere su importancia en la biogeoquímica de sus comunidades vegetales y muy probablemente de igual manera en otras regiones subtropicales y áridas del país donde abundan.

$\mathrm{Al}$ menos en algunas especies, la menor eficiencia de reabsorción de nutrientes pudo haber sido consencuencia de un mecanismo activo durante la senescencia. En algunas especies se observó un aumento en la concentración de P durante la senescencia. El incremento de nutrientes en la hoja senescente y la consecuente disminución en la eficiencia de reabsorción, particularmente en el caso del $\mathrm{P}$, suele suceder en sitios donde hay una gran cantidad de formas disponibles en el suelo (Milla et al. 2006). Otros han sugerido que este puede ser un mecanismo de conservación del nutriente en sitios en donde su disponibilidad edáfica es escasa (Pugnaire \& Chapin 1993). Es importante reconocer que al ser mayor la concentración de $\mathrm{N} \mathrm{y}$ $\mathrm{P}$ en hojas senescentes de las especies y tipos funcionales en el sitio de mayor aridez, permitiría reforzar su papel e importancia como nodrizas y facilitadoras, tal como se ha propuesto con anterioridad (Bertness \& Callaway 1994).

La menor eficiencia y suficiencia de reabsorción en el extremo más árido del gradiente pudiera considerarse como una estrategia de las plantas para mantener niveles de facilitación en el funcionamiento de la microbiota del suelo, su sobrevivencia y crecimiento, y mayores tasas de descomposición, mineralización y eventual disponibilidad (Zechmeister-Boltenstern et al. 2015). Como consecuencia, la hojarasca en este sitio, al ser rica en nutrientes, tenderá a descomponerse más fácilmente, aumentar la mate- ria orgánica y la fertilidad del suelo, e impactar de manera positiva la biogeoquímica del ecosistema (Austin 2011). El papel facilitador (Callaway 1995, Franklin et al. 2016) y la posible diferenciación de nichos biogeoquímicos en las plantas (Peñuelas et al. 2008, 2019), deberá ser explorado con mayor detalle, pues aquí encontramos que esto puede estar sucediendo.

Estequiometría ecológica y reabsorción. El cociente estequiométrico foliar cambió durante el proceso de reabsorción. El cociente brinda una idea de la fisiología de las especies en el medio que habitan y de su nicho biogeoquímico (Peñuelas et al. 2008, 2019). El cociente C:N en la hoja verde hace referencia al tiempo de vida y la tasa fotosintética, mientras que la relación $\mathrm{N}: \mathrm{P}$ y C:P están relacionadas al crecimiento (Vrede et al. 2004). Las diferencias en el incremento de las proporciones de $\mathrm{C}: \mathrm{N}$ en las hojas senescentes, sugiere que una mayor concentración de $\mathrm{N}$ fue solubilizado y reabsorbido en los sitios menos áridos, mientras que el $\mathrm{C}$, al formar parte de moléculas de difícil hidrólisis se reabsorbe en menor cantidad (McGroddy et al. 2004). Una consecuencia de las altas proporciones de $\mathrm{C}: \mathrm{N}$ en las hojas senescentes es la baja calidad en la hojarasca (Aerts 1997, Melillo et al. 1982), lo que puede sugerir una lenta descomposición en los sitios menos áridos ( $\mathrm{CH}$ y SR), así como mayor calidad y tasas de descomposición en el sitio más árido (SJ), dado su menor cociente.

En contraste, el cociente N:P disminuyó en las hojas senescentes respecto de las hojas verdes al compararse en todos los sitios. Las diferencias estequiométricas en N:P no fueron significativas entre los extremos del gradiente (SJ y $\mathrm{CH}$ ), pero sí mayor en el sitio intermedio (SR) (Figura $\underline{3 b})$. El menor promedio en N:P durante la senescencia indica que el $\mathrm{N}$ fue proporcionalmente más reabsorbido que el $\mathrm{P}$, aun cuando su reabsorción aumentó en promedio en especies de los sitios menos áridos, como lo indica la mayor proporción C:P de hojas senescentes (Figura 3c). Una característica común fue la gran variabilidad en los cocientes estequiométricos en todos los sitios, lo que sugiere la diversidad en las respuestas que pueden ejercer los individuos y las especies sobre los procesos biogeoquímicos del suelo a lo largo de un gradiente de aridez.

La eficiencia y suficiencia de reabsorción modificaron los patrones estequiométricos que se ven reflejados en las concentraciones de la hoja senescente y posteriormente en la hojarasca, de manera destacada en las leguminosas (aquí diferenciada como tipo funcional), que parecen no responder a estrategias de conservación de recursos. 
Las diferencias encontradas durante la senescencia entre los tipos funcionales, en particular en las leguminosas, afectaron la estequiometría de la hojarasca, lo que deberá influir en los procesos biogeoquímicos de los sitios. Lo anterior significa que el comportamiento de los tipos funcionales dominantes en las comunidades, influirá de manera importante en la magnitud y dirección de futuros desacoplamientos estequiométricos de diferentes niveles tróficos, tal como ha sido descrito en relación al incremento de aridez regional debido al cambio climático global (Delgado-Baquerizo et al. 2017).

Apenas empezamos a vislumbrar la gran importancia de la integración entre las respuestas de reabsorción y la estequiometría ecológica en la porción verde (por encima del suelo, plantas y relaciones planta-herbívoros) y café (bajo el suelo, hojarasca-comunidades microbianas y micorrízicas-suelo), por lo que deberán ser prioritarios mayor número de estudios en estos temas, incluyendo a las especies, tipos funcionales y ecosistemas áridos y semiáridos subtropicales del país y el mundo.

\section{Agradecimientos}

Este manuscrito mejoró sustancialmente con las atinadas sugerencias del editor y dos revisores anónimos, así como de D.M. Acuña. MGAM agradece el apoyo a CONACYT y Posgrado en Biociencias por la beca durante sus estudios de Maestría. AEC agradece el apoyo de CONACYT (CB61865 y CB223525) y CONABIO (LI005). AEC, JRLL y JMLIS agradecen a la Universidad de Sonora por su continuo apoyo. Agradecemos a D.M. Acuña y K. Campos, y el Laboratorio de Ecofisiología Vegetal por su apoyo en campo y laboratorio, y a los Srs. Luis Sierra Maldonado (Rancho el Churi), Juan Coronado Ballesteros (Shangai-Sta. Rosalía) y Alejandro Morales Aguilar (Rancho San Judas) por permitirnos el uso de sus instalaciones para realizar este trabajo de investigación.

\section{Literatura citada}

Adams MA, Turnbull TL, Sprent JI, Buchmann N. 2016. Legumes are different: Leaf nitrogen, photosynthesis, and water use efficiency. Proceedings of the $\mathrm{Na}$ tional Academy of Sciences of the United States of America 113: 4098-4103. DOI: https://doi.org/10.1073/ pnas. 1523936113

Aerts R. 1996. Nutrient resorption from senescing leaves of perennials: are there general patterns? Journal of Ecology 84: 597-608. DOI: https://doi.org/10.2307/2261481
Aerts R. 1997. Nitrogen partitioning between resorption and decomposition pathways: a trade-off between nitrogen use efficiency and litter decomposibility? Oikos 80: 603-606. DOI: https://doi.org/10.2307/3546636

Austin AT. 2011. Has water limited our imagination for aridland biogeochemistry? Trends in Ecology and Evolution 26: 229-235. DOI: https://doi.org/10.1016/j. tree.2011.02.003

Bertiller MB, Sain CL, Carrera A, Vargas D. 2005. Patterns of nitrogen and phosphorus conservation in dominant perennial grasses and shrubs across an aridity gradient in Patagonia, Argentina. Journal of Arid Environments 62: 209-223. DOI: https://doi.org/10.1016/j. jaridenv.2004.11.011

Bertness MD, Callaway RM. 1994. Positive interactions in communities. Trends in Ecology and Evolution 9: 191-193. DOI: https://doi.org/10.1016/0169$\underline{5347(94) 90088-4}$

Bloom AJ, Chapin FS, Mooney HA. 1985. Resource limitation in plants- an economic analogy. Annual Review of Ecology and Systematics 16: 363-392. DOI: https:// doi.org/10.1146/annurev.es.16.110185.002051

Bonfante P, Genre A. 2010. Mechanisms underlying beneficial plant-fungus interactions in mycorrhizal symbiosis. Nature Communications 1: 48. DOI: https://doi. org/10.1038/ncomms 1046

Brant AN, Chen HYH. 2015. Patterns and mechanisms of nutrient resorption in plants. Critical Reviews in Plant Sciences 34: 471-486. DOI: https://doi.org/10.1080/07 $\underline{352689.2015 .1078611}$

Callaway RM. 1995. Positive interactions among plants. Botanical Review 61: 306-349. DOI: https://doi. org/10.1007/BF02912621

Campanella MV, Bertiller MB. 2011. Is N-resorption efficiency related to secondary compounds and leaf longevity in coexisting plant species of the arid Patagonian Monte, Argentina? Austral Ecology 36: 395-402. DOI: https://doi.org/10.1111/j.1442-9993.2010.02165.x

Castellanos AE, Bravo LC, Koch GW, Llano J, López D, Méndez R, Rodríguez JC, Romo R, Sisk TD, YanesArvayo G. 2010. Impactos ecológicos por el uso del terreno en el funcionamiento de ecosistemas áridos y semiáridos. In: Molina FE, Van Devender TR, eds. Diversidad Biológica de Sonora. México: Universidad Nacional Autónoma de México - Comisión Nacional para el Conocimiento y Uso de la Biodiversidad, 157186. ISBN: 978-607-02-0427-2

Castellanos AE, Llano-Sotelo JM, Machado-Encinas LI, López-Piña JE, Romo-Leon JR, Sardans J, Peñuelas 
J. 2018. Foliar C, N, and P stoichiometry characterize successful plant ecological strategies in the Sonoran Desert. Plant Ecology 219: 775-788. DOI: https://doi. org/10.1007/s11258-018-0833-3

Celaya-Michel H, García-Oliva F, Rodríguez JC, Castellanos-Villegas AE. 2015. Cambios en el almacenamiento de nitrógeno y agua en el suelo de un matorral desértico transformado a sabana de buffel (Pennisetum ciliare (L.) Link). Terra Latinoamericana 33: 79-93.

Chapin III FS, Kedrowski RA. 1983. Seasonal changes in nitrogen and phosphorous fractions and autumn retranslocation in evergreen and deciduous Taiga trees. Ecology 64: 376-391. DOI: https://doi.org/10.2307/1937083

Chapin III FS, Moilanen L. 1991. Nutritional controls over nitrogen and phosphorus resorption from Alaskan birch leaves. Ecology 72: 709-715. DOI: https://doi. org/10.2307/2937210

Chávez-Vergara BM, González-Rodríguez A, Etchevers JD, Oyama K, García-Oliva F. 2015. Foliar nutrient resorption constrains soil nutrient transformations under two native oak species in a temperate deciduous forest in Mexico. European Journal of Forest Research 134: 803-817. DOI: https://doi.org/10.1007/s10342-0150891-1

Chávez-Vergara BM, Merino A, González-Rodríguez A, Oyama K, García-Oliva F. 2018. Direct and legacy effects of plant-traits control litter decomposition in a deciduous oak forest in Mexico. PeerJ 6: e5095. DOI: https://doi.org/10.7717/peerj.5095

CONAGUA [Comisión Nacional del Agua]. 2019. Climogramas 1981-2010. https://smn.conagua.gob.mx/es/ climatologia/informacion-climatologica/climogramas-1981-2010 (accessed november 10, 2018).

Cornelissen JHC. 1996. An experimental comparison of leaf decomposition rates in a wide range of temperate plant species and types. Journal of Ecology 84: 573582. DOI: https://doi.org/10.2307/2261479

Côté B, Fyles JW, Djalilvand H. 2002. Increasing N and $\mathrm{P}$ resorption efficiency and proficiency in northern deciduous hardwoods with decreasing foliar $\mathrm{N}$ and $\mathrm{P}$ concentrations. Annals of Forest Science 59: 275-281. DOI: https://doi.org/10.1051/forest:2002023

Cross AF, Schlesinger WH. 1999. Plant regulation of soil nutrient distribution in the northern Chihuahuan Desert. Plant Ecology 145: 11-25. DOI: https://doi. org/10.1023/A:1009865020145

Delgado-Baquerizo M, Eldridge DJ, Maestre FT, Ochoa V, Gozalo B, Reich PB, Singh BK. 2017. Aridity decouples $\mathrm{C}: \mathrm{N}: \mathrm{P}$ stoichiometry across multiple trophic levels in terrestrial ecosystems. Ecosystems 21: 459468. DOI: https://doi.org/10.1007/s10021-017-0161-9

Drenovsky RE, Pietrasiak N, Short TH. 2019. Global temporal patterns in plant nutrient resorption plasticity. Global Ecology and Biogeography 28: 728-743. DOI: https://doi.org/10.1111/geb.12885

Eckstein RL, Karlsson PS, Weih M. 1999. Leaf life span and nutrient resorption determinants of plant nutrient conservation in temperate-arctic regions. New Phytologist 143: 177-189. DOI: https://doi.org/10.1046/j.14698137.1999.00429.x

Franklin KA, Sommers PN, Aslan CE, López BR, Bronstein JL, Bustamante E, Búrquez A, Medellín RA, Marazzi B. 2016. Plant biotic interactions in the Sonoran Desert: Current knowledge and future research perspectives. International Journal of Plant Sciences 177: 217-234. DOI: https://doi.org/10.1086/684261

Franklin O, Ågren GI. 2002. Leaf senescence and resorption as mechanisms of maximizing photosynthetic production during canopy development at $\mathrm{N}$ limitation. Functional Ecology 16: 727-733. DOI: https://doi. org/10.1046/j.1365-2435.2002.00674.X

Freschet GT, Cornwell WK, Wardle DA, Elumeeva TG, Liu W, Jackson BG, Onipchenko VG, Soudzilovskaia NA, Tao J, Cornelissen JH. 2013. Linking litter decomposition of above- and below-ground organs to plant-soil feedbacks worldwide. Journal of Ecology 101: 943-952. DOI: https://doi.org/10.1111/1365$\underline{2745.12092}$

Gerdol R, Iacumin P, Marchesini R, Bragazza L. 2000. Water- and nutrient-use efficiency of a deciduous species, Vaccinium myrtillus, and an evergreen species, V. vitis-idaea, in a subalpine dwarf shrub heath in the southern Alps, Italy. Oikos 88: 19-32. DOI: https://doi. org/10.1034/j.1600-0706.2000.880104.x

Güsewell S. 2004. N: P ratios in terrestrial plants: variation and functional significance. New Phytologist 164: 243-266. DOI: https://doi.org/10.1111/j.14698137.2004.01192.x

Güsewell S. 2005. Nutrient resorption of wetland graminoids is related to the type of nutrient limitation. Functional Ecology 19: 344-354. DOI: https://doi. org/10.1111/j.0269-8463.2005.00967.x

Güsewell S, Gessner MO. 2009. N: P ratios influence litter decomposition and colonization by fungi and bacteria in microcosms. Functional Ecology 23: 211-219. DOI: https://doi.org/10.1111/j.1365-2435.2008.01478.x

Han W, Fang J, Guo D, Zhang Y. 2005. Leaf nitrogen and phosphorus stoichiometry across 753 terrestrial plant 
species in China. New Phytologist 168: 377-385. DOI: https://doi.org/10.1111/j.1469-8137.2005.01530.x

He H, Bleby TM, Veneklaas EJ, Lambers H. 2011. Dinitrogen-fixing Acacia species from phosphorus-impoverished soils resorb leaf phosphorus efficiently. Plant Cell and Environment 34: 2060-2070. DOI: http://doi. org/10.1111/j.1365-3040.2011.02403.x

Hinojo-Hinojo C, Castellanos AE, Huxman T, Rodriguez JC, Vargas R, Romo-León JR, Biederman JA. 2019. Native shrubland and managed buffelgrass savanna in drylands: Implications for ecosystem carbon and water fluxes. Agricultural and Forest Meteorology 268: 269-278. DOI: https://doi.org/10.1016/j. agrformet.2019.01.030

Killingbeck KT. 1996. Nutrients in senesced leaves: keys to the search for potential resorption and resorption proficiency. Ecology 77: 1716-1727. DOI: https://doi. org/10.2307/2265777

Kobe RK, Lepczyk CA, Iyer M. 2005. Resorption efficiency decreases with increasing green leaf nutrients in a global data set. Ecology 86: 2780-2792. DOI: https:// doi.org/10.1890/04-1830

Martínez DE, Costa ML, Guiamet JJ. 2008. Senescenceassociated degradation of chloroplast proteins inside and outside the organelle. Plant Biology 10: 15-22. DOI: https://doi.org/10.1111/j.1438-8677.2008.00089.x

McGroddy ME, Daufresne T, Hedin LO. 2004. Scaling of $\mathrm{C}: \mathrm{N}: \mathrm{P}$ stoichiometry in forests worldwide: Implications of terrestrial redfield-type ratios. Ecology 85: 2390-2401. DOI: https://doi.org/10.1890/03-0351

Melillo JM, Aber JD, Muratore JF. 1982. Nitrogen and lignin control of hardwood leaf litter decomposition dynamics. Ecology 63: 621-626. DOI: https://doi. org/10.2307/1936780

Milla R, Palacio-Blasco S, Maestro-Martínez M, Montserrat-Martí G. 2006. Phosphorus accretion in old leaves of a Mediterranean shrub growing at a phosphorus-rich site. Plant and Soil 280: 369-372. DOI: https://doi. org/10.1007/s11104-005-3529-0

Mooshammer M, Wanek W, Schnecker J, Wild B, Leitner S, Hofhansl F, Blöchl A, Hämmerle I, Frank AH, Fuchslueger L, Keiblinger KM, Zechmeister-Boltenstern S, Richter A. 2011. Stoichiometric controls of nitrogen and phosphorus cycling in decomposing beech leaf litter. Ecology 93: 770-782. DOI: https://doi. org/10.1890/11-0721.1

Mooshammer M, Wanek W, Zechmeister-Boltenstern S, Richter AA. 2014. Stoichiometric imbalances between terrestrial decomposer communities and their resourc- es: mechanisms and implications of microbial adaptations to their resources. Frontiers in Microbiology 5: 22. DOI: https://doi.org/10.3389/fmicb.2014.00022

Murphy J, Riley JP. 1962. A modified single solution method for the determination of phosphate in natural waters. Analytica Chimica Acta 27: 31-36. DOI: https://doi.org/10.1016/S0003-2670(00)88444-5

Peñuelas J, Fernández-Martínez M, Ciais P, Jou D, Piao S, Obersteiner M, Vicca S, Janssens IA, Sardans J. 2019. The bioelements, the elementome, and the biogeochemical niche. Ecology 100: e02652. DOI: https:// doi.org/10.1002/ecy.2652

Peñuelas J, Sardans J, Ogaya R, Estiarte M. 2008. Nutrient stoichiometric relations and biogeochemical niche in coexisting plant species: effect of simulated climate change. Polish Journal of Ecology 56: 613-622.

Prieto I, Querejeta JI. 2020. Simulated climate change decreases nutrient resorption from senescing leaves. Global Change Biology 26: 1795-1807. DOI: https:// doi.org/10.1111/gcb.14914

Pugnaire FI, Chapin III FS. 1993. Controls over nutrient resorption from leaves of evergreen mediterranean species. Ecology 74: 124-129. DOI: https://doi. org/10.2307/1939507

Reed SC, Townsend AR, Davidson EA, Cleveland CC. 2012. Stoichiometric patterns in foliar nutrient resorption across multiple scales. New Phytologist 196: 173-80. DOI: https://doi.org/10.1111/j.14698137.2012.04249.x

Schlesinger WH, Pilmanis AM. 1998. Plant-soil interactions in deserts. In: Van Breemen N, ed. Plant-Induced Soil Changes: Processes and feedbacks. Developments in Biogeochemistry, Vol. 4. Dordrecht: Springer; 169187. DOI: https://doi.org/10.1007/978-94-017-2691-7 9

Schlesinger WH, Raikes JA, Hartley AE, Cross AF. 1996. On the spatial pattern of soil nutrients in desert ecosystems. Ecology 77: 364-374. DOI: https://doi. org/10.2307/2265615

See CR, Yanai RD, Fisk MC, Vadeboncoeur MA, Quintero BA, Fahey TJ. 2015. Soil nitrogen affects phosphorus recycling: foliar resorption and plant-soil feedbacks in a northern hardwood forest. Ecology 96: 2488-2498. DOI: https://doi.org/10.1890/15-0188.1

Shreve F, Wiggins IL. 1964. Vegetation and Flora of the Sonoran Desert. Stanford: Stanford University Press. ISBN: 9780804701631

Sohrt J, Herschbach C, Weiler M. 2018. Foliar P- but not $\mathrm{N}$ resorption efficiency depends on the $\mathrm{P}$-concentration and the N:P ratio in trees of temperate forests. Trees 
32: 1443-1455. DOI: https://doi.org/10.1007/s00468018-1725-9

Sterner RW, Elser JJ. 2002. Ecological Stoichiometry: The Biology of Elements from Molecules to the Biosphere. Princeton: Princeton University Press. ISBN: 978-0691-07491-7

Stewart JR, Kennedy GJ, Landes RD, Dawson JO. 2008. Foliar-nitrogen and phosphorus resorption patterns differ among nitrogen-fixing and nonfixing temperate-deciduous trees and shrubs. International Journal of Plant Sciences 169: 495-502. DOI: https://doi.org/10.1086/528749

Tateno M. 2003. Benefit to N2-fixing alder of extending growth period at the cost of leaf nitrogen loss without resorption. Oecologia 137: 338-343. DOI: https://doi. org/10.1007/s00442-003-1357-6

Tully KL, Wood TE, Schwantes AM, Lawrence D. 2013. Soil nutrient availability and reproductive effort drive patterns in nutrient resorption in Pentaclethra macroloba. Ecology 94: 930-940. DOI: https://doi.org/10.1890/12-0781.1

Turner RM, Bowers JE, Burgess TL, Hastings JR. 1995. Sonoran Desert Plants: An Ecological Atlas. Tucson: University of Arizona Press. ISBN: 9780816515325.

Van Heerwaarden LM, Toet S, Aerts R. 2003. Current measures of nutrient resorption efficiency lead to a substantial underestimation of real resorption efficiency: facts and solutions. Oikos 101: 664-669. DOI: https:// doi.org/10.1034/j.1600-0706.2003.12351.x

Vergutz L, Manzoni S, Porporato A, Novais RF, Jackson RB. 2012. Global resorption efficiencies and concentrations of carbon and nutrients in leaves of terrestrial plants. Ecological Monographs 82: 205-220. DOI: https://doi.org/10.1890/11-0416.1

Vrede T, Dobberfuhl DR, Kooijman SALM, Elser JJ. 2004. Fundamental connections among organism $\mathrm{C}: \mathrm{N}: \mathrm{P}$ stoichiometry, macromolecular composition, and growth. Ecology 85: 1217-1229. DOI: https://doi. org/10.1890/02-0249

\footnotetext{
Editor de sección: Numa Pavón

Contribución de los autores: MGMA. diseño, trabajo de campo, análisis y escritura del manuscrito; AEC, diseño, trabajo de campo, análisis y escritura del manuscrito; JML1S. asesoría, análisis de nutrientes y base de datos, revisión y aportación al documento; JRRL, asesoría, revisión y aportación al documento; KCC. asesoría, revisión y aportación al documento; ME, asesoría, revisión y aportación al documento.
}

West NE, Skujins J. 1978. Nitrogen in Desert Ecosystems. Stroudsburg: Dowden, Hutchinson \& Ross. ISBN: 0879333332.

Wright IJ, Westoby M. 2002. Leaves at low versus high rainfall: coordination of structure, lifespan and physiology. New Phytologist 155: 403-416. DOI: https://doi. org/10.1046/j.1469-8137.2002.00479.x

Wright IJ, Westoby M. 2003. Nutrient concentration, resorption and lifespan: leaf traits of Australian sclerophyll species. Functional Ecology 17: 10-19. DOI: https://doi.org/10.1046/j.1365-2435.2003.00694.X

Yuan Z-Y, Chen HYH. 2009. Global-scale patterns of nutrient resorption associated with latitude, temperature and precipitation. Global Ecology and Biogeography 18: 11-18. DOI: https://doi.org/10.1111/j.14668238.2008.00425.X

Yuan Z-Y, Chen HYH. 2015. Decoupling of nitrogen and phosphorus in terrestrial plants associated with global changes. Nature Climate Change 5: 465-469. DOI: https://doi.org/10.1038/nclimate2549

Yuan Z-Y, Li L-H, Han X-G, Huang J-H, Jiang G-M, Wan S-Q, Zhang W-H, Chen Q-S. 2005. Nitrogen resorption from senescing leaves in 28 plant species in a semiarid region of northern China. Journal of Arid Environments 63: 191-202. DOI: https://doi.org/10.1016/j. jaridenv.2005.01.023

Zechmeister-Boltenstern S, Keiblinger KM, Mooshammer M, Peñuelas J, Richter A, Sardans J, Wanek W. 2015. The application of ecological stoichiometry to plant-microbial-soil organic matter transformations. Ecological Monographs 85: 133-155. DOI: https://doi. org/10.1890/14-0777.1

Zhao G, Shi P, Wu J, Xiong D, Zong N, Zhang X. 2017. Foliar nutrient resorption patterns of four functional plants along a precipitation gradient on the Tibetan Changtang Plateau. Ecology and Evolution 7: 72017212. DOI: https://doi.org/10.1002/ece3.3283 